\title{
Prevalência e fatores associados ao sobrepeso/obesidade e à hipertensão arterial sistêmica em crianças da rede privada de ensino de Divinópolis/MG
}

\author{
Prevalence and factors associated with \\ overweight/obesity and systemic arterial hypertension in the \\ private education network children of Divinópolis, MG
}

Silvana Diniz Ferreira¹, Fábio Peron Carballo², Fabrízio Furtado de Sousa², Denise Maria Rover da Silva ${ }^{3}$

\begin{abstract}
Resumo
Este trabalho teve como objetivo investigar a prevalência de sobrepeso/obesidade e da hipertensão arterial sistêmica (HAS) em crianças, com idade entre 8 a 10 anos, da rede privada de ensino de Divinópolis, em Minas Gerais, e os fatores de risco associados. Foram mensurados os níveis de pressão arterial (PA), peso corporal e altura das crianças. Os fatores de risco foram obtidos por meio de questionário. Os dados foram analisados no software STATA e a significância adotada foi de $p<0,05$. Foram amostradas 199 crianças (107 meninas e 92 meninos), das quais 33,2\% apresentaram sobrepeso/obesidade. $O$ excesso de peso mostrou-se associado ao gênero e ao peso da mãe. Com relação à HAS, 33,2\% das crianças apresentaram PA considerada como limítrofe ou elevada. Os níveis de PA alterados mostraram-se relacionados à raça e ao tempo de aleitamento materno. Outra característica associada aos níveis pressóricos alterados foi o excesso de peso corpóreo, reforçando os dados da literatura que demonstram uma importante associação entre HAS e sobrepeso/obesidade.
\end{abstract}

Palavras-chave: obesidade; hipertensão arterial sistêmica; crianças.

\begin{abstract}
This study aimed to investigate the prevalence of overweight/obesity and systemic arterial hypertension (SAH) in children aged 8-10 years of private schools in Divinópolis, MG and the associated risk factors. Levels of blood pressure, body weight and height of children were measured. The risk factors were obtained by questionnaire. Data were analyzed using STATA software and statistical significance was set at $p<0.05$. Sampling included 199 children (107 girls and 92 boys). Of these, 33.2\% were overweight/obese. Overweight was associated with the gender and mother's weight. Regarding hypertension, $33.2 \%$ of children showed borderline or high blood pressure. The altered levels of blood pressure proved to be related to race and breastfeeding duration. Another feature associated with altered blood pressure was excess body weight, reinforcing the literature data that demonstrate a significant association between hypertension and overweight/obesity.
\end{abstract}

Keywords: obesity; hypertension; children.

'Graduada em Ciências Biológicas pela Fundação Educacional de Divinópolis (FUNEDI), Universidade Estadual de Minas Gerais (UEMG) - Divinópolis (MG), Brasil.

${ }_{2}^{2}$ Professor Mestre da Fundação Educacional de Divinópolis (FUNEDI), Universidade Estadual de Minas Gerais (UEMG) - Divinópolis (MG), Brasil. ${ }_{3}^{3}$ Professora Doutora da Fundação Educacional de Divinópolis (FUNEDI), Universidade Estadual de Minas Gerais (UEMG) - Divinópolis (MG), Brasil. Trabalho realizado na Fundação Educacional de Divinópolis (FUNEDI), Universidade Estadual de Minas Gerais (UEMG) - Divinópolis (MG), Brasil. Endereço para correspondência: Denise Maria Rover Silva - Av. Paraná, 3001 - Jardim Belvedere II - CEP: 35501-170 - Divinópolis (MG), Brasil Email:drover@divinopolisuemg.com.br

Fonte de financiamento: 0 presente estudo recebeu suporte financeiro da FAPEMIG.

Conflito de interesses: nada a declarar. 


\section{INTRODUÇÃO}

Atualmente, a obesidade é considerada um problema de saúde pública em todo o mundo. No Brasil, entre os indivíduos adultos, $49,9 \%$ da população apresenta excesso de peso, dos quais $14,8 \%$ possuem obesidade ${ }^{1}$ Além das questões estéticas relacionadas ao excesso de peso, a obesidade é um fator de risco para uma série de doenças, como o diabetes e a hipertensão arterial sistêmica (HAS) ${ }^{2}$.

Apesar de mais vastamente estudada as consequências da obesidade em adultos, o excesso de peso corpóreo também traz importantes implicações à saúde das crianças ${ }^{3}$. Segundo Daniels ${ }^{3}$, a obesidade infantil vem aumentando em todas as faixas etárias e contribuindo na promoção de vários agravos à saúde, na infância e na idade adulta. No Brasil, entre crianças com idade menor que 5 anos, a prevalência de sobrepeso/obesidade é de 7,5\% e torna-se ainda mais preocupante na faixa etária subsequente (5 a 9 anos), na qual atinge 33,5\% das crianças ${ }^{1}$. Vários estudos têm demonstrado que o excesso de peso corpóreo é um dos principais determinantes da elevação da pressão arterial (PA) em crianças, sobretudo a partir dos 5 anos de idade, com uma relação direta entre índice de massa corpórea (IMC), níveis de PA e mortalidade por doenças cardiovascular. Sorof e Daniels ${ }^{5}$, crianças obesas apresentam probabilidade três vezes maior de desenvolver HAS, quando comparadas às crianças não obesas.

Mesmo que as consequências do excesso de peso não se expressem durante a infância, vários estudos têm demonstrado que a HAS do adulto é uma doença que se inicia na infância ${ }^{6,7}$.

A HAS é uma síndrome multifatorial caracterizada pela presença de níveis tensionais elevados, associados a alterações metabólicas, hormonais e fenômenos tróficos, como hipertrofia cardíaca e vascular ${ }^{6}$. A elevação e a manutenção de níveis pressóricos alterados aumentam o risco de doenças cardiovasculares, das quais a HAS é reconhecida como o principal fator de risco para a morbidade e mortalidade precoce ${ }^{6,7}$.

No Brasil, a prevalência de HAS na população adulta varia de 22,3 a $43,9 \%{ }^{8}$, mas entre crianças, não há inquéritos epidemiológicos que evidenciem esse problema. Porém diversos estudos locais têm demonstrado prevalências de HAS que variam de 2,5 a 44,7\% em crianças, dependendo da metodologia utilizada?.

Divinópolis é designada cidade polo da Macrorregional Oeste de Minas Gerais, com população estimada em 226.345 habitantes ${ }^{1}$. Uma característica da cidade é a expectativa de vida da população, que vive em média 75,5 anos, considerada maior que a média nacional $\left(73,4\right.$ anos) e estadual $(75,1 \text { anos })^{1}$. Esse aspecto da longevidade tem como fator importante na assistência à saúde o fato de que pessoas envelhecidas têm maior predisposição às doenças crônicas, como os problemas circulatórios, que atualmente representam a principal causa de morte no município $^{10}$. Considerando que a obesidade e a HAS, quando desenvolvidas na infância, apresentam grande probabilidade de se manterem durante a fase adulta e que são importantes fatores de predisposição para doenças cardiovasculares, consideramos que o levantamento de dados acerca de sua prevalência em crianças e de seus fatores relacionados é de suma importância.

Recentemente, um estudo realizado na cidade avaliou o estado nutricional de crianças, com idade entre 6 e 14 anos, matriculadas em escolas públicas, encontrando prevalências de 10,7 e 6,43\% para sobrepeso e obesidade, respectivamente ${ }^{11}$. Apesar desses achados, vários trabalhos na literatura sugerem que, em países em desenvolvimento, a prevalência de sobrepeso/obesidade é maior na população mais favorecida economicamente ${ }^{12}$. Dessa forma, consideramos que o estudo dos fatores relacionados a essas alterações em crianças de maior nível socioeconômico, como as que se encontram matriculadas em escolas privadas, mostra-se prioritário, pois a divulgação deles pode favorecer a adoção de medidas de prevenção que beneficiarão um maior número de crianças afetadas pelo sobrepeso/obesidade.

\section{METODOLOGIA}

O presente estudo foi desenvolvido entre dezembro de 2011 e novembro de 2012, em 9 das 13 escolas da rede privada de ensino da cidade de Divinópolis/MG que oferecem o ensino fundamental I. Todas as escolas cujos diretores concordaram com a realização do estudo foram pesquisadas.

Antes da obtenção dos dados, os responsáveis pelas crianças receberam um termo de consentimento livre e esclarecido (TCLE), por meio do qual foram informados sobre a realização e os objetivos do estudo. Somente foram analisadas as crianças cujos pais concordaram com a realização do estudo.

Para a coleta dos dados, todas as crianças, com idades entre 8 e 10 anos, matriculadas nas escolas privadas que concordaram com a realização do estudo foram analisadas. Os estudantes que se recusaram a participar da pesquisa ou não estiveram presentes nas datas de coleta de dados foram excluídos do estudo. Foram mensurados os níveis de PA, peso corporal e altura das crianças.

Os demais dados, como idade, sexo, raça, peso ao nascer, tempo de gestação, tempo de amamentação, prática de atividade física, horas diárias na frente da TV, consumo de fast food, escolaridade e peso da mãe e renda familiar foram obtidos por meio de questionário próprio, encaminhado aos pais dos alunos participantes.

Com relação às variáveis testadas, a raça das crianças pôde ser declarada como branca, parda ou negra, com as duas últimas agrupadas para análise. Para classificação do peso ao nascer, utilizou-se o critério da Organização Mundial da Saúde (OMS): baixo peso (crianças com menos de $2.500 \mathrm{~g}$ ), peso insuficiente (2.500 g a $2.999 \mathrm{~g}$ ), peso adequado (3.000 g a $3.999 \mathrm{~g})$, com o agrupamento dos dois últimos para análise, e excesso de 
peso (4.000 g ou mais) ${ }^{13}$. Com relação ao tempo de gestação, foi considerado prematuro crianças que nasceram com idade gestacional inferior a 37 semanas $^{13}$. Para avaliação do peso das mães, foi calculado o IMC por meio da relação peso $(\mathrm{kg}) / \mathrm{altura}^{2}$ (m), utilizando-se quatro categorias para classificação: baixo peso (IMC<19,8), peso normal (IMC entre 19,8 e 26,0), sobrepeso (IMC entre 26,0 e 29,0) e obesidade (IMC $\geq 29,0$ ), com os dois últimos agrupados para análise. Como prática de atividade física, foi considerada a realização de qualquer atividade esportiva exercida regularmente, no mínimo três vezes por semana, com pelo menos 1 hora de duração diária.

O presente estudo foi aprovado pelo Comitê de Ética e Pesquisa da Fundação Educacional de Divinópolis, Universidade Estadual de Minas Gerais (FUNEDI/UEMG) (Parecer n. 27/2011).

\section{Avaliação antropométrica}

Para avaliar a composição corporal das crianças, foi utilizada a antropometria ${ }^{14}$.

Neste trabalho, os índices antropométricos das crianças foram obtidos a partir da combinação de quatro dados: peso, sexo, idade e altura ${ }^{15}$. Para isso, utilizou-se o programa WHO anthroplus, versão 1.0.3.

Neste estudo, foi empregado o escore- $z$, considerando como eutrófico os valores de -1, 0, 1; o que estivesse abaixo desses valores $(<-1)$ foi considerado como baixo peso, e acima, como sobrepeso $(>1)$ e obesidade $(>2)^{15}$.

\section{Avaliação do peso corporal, altura e aferição da pressão arterial}

O peso corporal das crianças foi obtido utilizando uma balança digital (capacidade de $180 \mathrm{~kg}$ ), e a estatura avaliada por meio um estadiômetro portátil de alumínio (com capacidade de 300 a $2.000 \mathrm{~mm}$ ).

Para a medida e avaliação da PA, foram seguidas as recomendações da V Diretrizes Brasileiras de Hipertensão Arterial ${ }^{8}$.

A PA foi verificada pelo método oscilométrico com aparelho monitor de PA automático (marca Omron, modelo HEM 742 INT) ${ }^{16}$. Foram utilizados manguitos Omron de tamanho apropriado à circunferência dos braços das crianças e realizadas duas medidas em dias diferentes - a menor das duas foi considerada no estudo.

Para a conversão dos valores de PA sistólica e diastólica em percentil, foi utilizada a classificação de PA na infância, de acordo com as V Diretrizes Brasileiras de Hipertensão Arterial ${ }^{8}$.

\section{Análise dos dados}

Para avaliar os possíveis fatores associados ao excesso de peso e à PA alterada, foi utilizado o modelo de Poisson com variâncias robustas, bi e multivariadas. Foram estimados os valores de razão de prevalência ( $\mathrm{RP}$ ) bivariados, com seu respectivo intervalo de confiança de 95\% (IC95\%). Também foi realizada a análise multivariada com o objetivo de controlar possíveis fatores de confusão. Foram selecionadas para entrar no modelo multivariado as variáveis que apresentaram valor-p $<0,20$ na análise bivariada. Para construção dos modelos, utilizou-se o critério backward. Para permanência das variáveis no modelo final, considerou-se um nível de significância de 5\%. Também foram estimados os valores de RP e seu IC95\% para cada modelo final. Foi utilizado o software STATA, versão 10.0.

\section{RESULTADOS}

\section{Análise do peso corpóreo e fatores associados}

Foram avaliadas 199 crianças, das quais 73 (36,6\%) apresentavam 8 anos de idade, 63 (31,7\%) tinham 9 anos e $63(31,7 \%), 10$ anos de idade.

Com relação ao peso corpóreo, 33,2\% das crianças apresentaram peso elevado, das quais 19,1\% tinham sobrepeso e $14,1 \%$ apresentaram obesidade. Uma maior prevalência de sobrepeso/obesidade foi observada entre os meninos $(44,6 \%)$ do que entre as meninas $(23,4 \%)$. Com relação à idade das crianças analisadas, não foi observada diferença significativa na prevalência de sobrepeso/obesidade (Tabela 1).

Outro fator que se mostrou associado ao excesso de peso das crianças foi o tempo em que elas permanecem em frente à TV. Crianças que permanecem por mais de 3 horas/diárias nessa atividade apresentaram maior prevalência de sobrepeso/obesidade $(\mathrm{p}<0,05)$.

Os demais dados analisados não demonstraram significância, quando relacionados ao peso corpóreo das crianças (Tabela 1).

\section{Análise dos níveis pressóricos e fatores associados}

Com relação à PA, 33,2\% das crianças analisadas apresentaram níveis pressóricos alterados, das quais $24,6 \%$ foram consideradas como limítrofes, e 8,6\%, como hipertensivas. Dentre as meninas, a prevalência de PA alterada foi de 30,8\%, e dentre os meninos, $35,9 \%$, não havendo diferença significativa. Também não foi observada diferença entre as idades das crianças e a prevalência de níveis pressóricos alterados (Tabela 2).

A raça das crianças e o tempo de amamentação apresentaram-se significativamente relacionados aos níveis pressóricos. Com relação à raça, crianças não brancas apresentaram maior prevalência de níveis pressóricos alterados $(45,7 \%)$ do que crianças brancas $(29,6 \%)(p<0,05)$. Com relação ao tempo de amamentação materna, crianças que não amamentaram demonstraram maior prevalência de níveis pressóricos alterados $(64,7 \%)$ do que crianças que amamentaram até seis meses $(41,2 \%)$ ou por mais tempo $(26,4 \%)(\mathrm{p}<0,01)$.

Os demais dados analisados não demonstraram relação significativa com os níveis pressóricos (Tabela 2). 
Tabela 1. Excesso de peso, segundo fatores relacionados, em crianças com idade entre 8 e 10 anos das escolas da rede privada de Divinópolis/MG

\begin{tabular}{|c|c|c|c|c|c|c|}
\hline \multirow{2}{*}{ Variável } & \multirow{2}{*}{$\frac{\text { Total }}{\mathbf{n}}$} & \multicolumn{2}{|c|}{ Excesso de peso } & \multicolumn{2}{|c|}{ Excesso de peso } & \multirow{2}{*}{ Valor $\mathbf{p}$} \\
\hline & & $\mathbf{n}$ & $\%$ & RP & (IC95\%) & \\
\hline \multicolumn{7}{|l|}{ Idade (anos) } \\
\hline 8 & 73 & 20 & 27,4 & 1,00 & - & 0,134 \\
\hline 9 & 63 & 27 & 42,9 & 1,56 & {$[0,98 ; 2,51]$} & \\
\hline 10 & 63 & 19 & 30,2 & 1,10 & {$[0,65 ; 1,87]$} & \\
\hline Total & 199 & 66 & 33,2 & & & \\
\hline \multicolumn{7}{|l|}{ Sexo } \\
\hline Feminino & 107 & 25 & 23,4 & 1,00 & - & 0,002 \\
\hline Masculino & 92 & 41 & 44,6 & 1,91 & {$[1,26 ; 2,88]$} & \\
\hline Total & 199 & 66 & 33,2 & & & \\
\hline Raça & & & & & & 0,634 \\
\hline Branca & 152 & 52 & 34,2 & 1,00 & - & \\
\hline Não branca & 46 & 14 & 30,4 & 0,89 & {$[0,54 ; 1,45]$} & \\
\hline Total & 198 & 66 & 33,3 & & & \\
\hline Peso ao nascer & & & & & & 0,617 \\
\hline Adequado & 124 & 44 & 35,5 & 1,00 & - & \\
\hline Baixo & 63 & 18 & 28,6 & 0,81 & {$[0,51 ; 1,27]$} & \\
\hline Excesso & 7 & 2 & 28,6 & 0,81 & {$[0,24 ; 2,67]$} & \\
\hline Total & 194 & 64 & 33,0 & & & \\
\hline Tempo gestação & & & & & & 0,984 \\
\hline Prematuro & 27 & 9 & 33,3 & 1,00 & - & \\
\hline Não prematuro & 170 & 57 & 33,5 & 0,99 & {$[0,56 ; 1,77]$} & \\
\hline Total & 197 & 66 & 33,5 & & & \\
\hline Tempo amamentação & & & & & & 0,052 \\
\hline 6 meses ou mais & 130 & 38 & 29,2 & 1,00 & - & \\
\hline Inferior a 6 meses & 51 & 18 & 35,3 & 1,20 & {$[0,76 ; 1,90]$} & \\
\hline Não amamentou & 17 & 10 & 58,8 & 2,00 & {$[1,23 ; 3,23]$} & \\
\hline Total & 198 & 66 & 33,3 & & & \\
\hline Atividade física ${ }^{\star}$ & & & & & & 0,556 \\
\hline Não pratica & 60 & 22 & 36,7 & 1,00 & - & \\
\hline Pratica & 136 & 44 & 32,4 & 0,88 & {$[0,58 ; 1,33]$} & \\
\hline Total & 196 & 66 & 33,7 & & & \\
\hline Horas TV/diária & & & & & & 0,041 \\
\hline Até 1 hora & 57 & 19 & 33,3 & 1,00 & - & \\
\hline Até 2 horas & 63 & 19 & 30,2 & 0,91 & {$[0,54 ; 1,54]$} & \\
\hline Até 3 horas & 49 & 12 & 24,5 & 0,74 & {$[0,40 ; 1,37]$} & \\
\hline Acima de 3 horas & 29 & 16 & 55,2 & 1,66 & {$[1,01 ; 2,72]$} & \\
\hline Total & 198 & 66 & 33,3 & & & \\
\hline Consumo de fast food & & & & & & 0,269 \\
\hline Não & 97 & 36 & 37,1 & 1,00 & - & \\
\hline Sim & 101 & 30 & 29,7 & 0,80 & {$[0,54 ; 1,19]$} & \\
\hline Total & 198 & 66 & 33,3 & & & \\
\hline Escolaridade da mãe & & & & & & 0,269 \\
\hline Fundamental & 19 & 5 & 26,3 & 1,00 & - & \\
\hline Médio & 85 & 24 & 28,2 & 1,07 & {$[0,47 ; 2,45]$} & \\
\hline Superior & 93 & 36 & 38,7 & 1,47 & {$[0,66 ; 3,26]$} & \\
\hline Total & 197 & 65 & 33,0 & & & \\
\hline Peso da mãe & & & & & & 0,064 \\
\hline Normal & 121 & 33 & 27,3 & 1,00 & - & \\
\hline Baixo peso & 4 & 2 & 50,0 & 1,83 & {$[0,66 ; 5,11]$} & \\
\hline Excesso & 67 & 29 & 43,3 & 1,59 & {$[1,06 ; 2,37]$} & \\
\hline Total & 192 & 66 & 34,4 & & & \\
\hline Renda familiar & & & & & & 0,720 \\
\hline Até R \$1.000,00 & 23 & 6 & 26,1 & 1,00 & - & \\
\hline Entre $\mathrm{R} \$ 1.000,00$ e $2.000,00$ & 58 & 19 & 32,8 & 1,26 & {$[0,57 ; 2,75]$} & \\
\hline Entre $\mathrm{R} \$ 2.000,00$ e $4.000,00$ & 118 & 41 & 34,7 & 1,33 & {$[0,64 ; 2,77]$} & \\
\hline Total & 199 & 66 & 33,2 & & & \\
\hline Pressão arterial da criança & & & & & & $<0,001$ \\
\hline Normal & 133 & 28 & 21,1 & 1,00 & - & \\
\hline Alterada & 66 & 38 & 57,6 & 2,73 & {$[1,85 ; 4,04]$} & \\
\hline Total & 199 & 66 & 33,2 & & & \\
\hline
\end{tabular}

*Foi considerada como prática de atividade física a realização de qualquer atividade esportiva exercida regularmente no mínimo, três vezes por semana, com pelo menos 1 hora de duração por dia 
Tabela 2. Pressão arterial alterada, segundo fatores relacionados, em crianças com idade entre 8 e 10 anos das escolas da rede privada de Divinópolis/MG

\begin{tabular}{|c|c|c|c|c|c|c|}
\hline \multirow{2}{*}{ Variável } & \multirow{2}{*}{$\frac{\text { Total }}{\mathbf{n}}$} & \multicolumn{2}{|c|}{ Pressão alterada } & \multicolumn{2}{|c|}{ Pressão alterada } & \multirow{2}{*}{ Valor $\mathbf{p}$} \\
\hline & & $\mathbf{n}$ & $\%$ & $\mathbf{R P}$ & (IC95\%) & \\
\hline \multicolumn{7}{|l|}{ Idade (anos) } \\
\hline 8 & 73 & 23 & 31,5 & 1,00 & - & 0,595 \\
\hline 9 & 63 & 19 & 30,2 & 0,96 & {$[0,58 ; 1,59]$} & \\
\hline 10 & 63 & 24 & 38,1 & 1,21 & {$[0,76 ; 1,92]$} & \\
\hline Total & 199 & 66 & 33,2 & & & \\
\hline Sexo & & & & & & 0,453 \\
\hline Feminino & 107 & 33 & 30,8 & 1,00 & - & \\
\hline Masculino & 92 & 33 & 35,9 & 1,16 & {$[0,78 ; 1,73]$} & \\
\hline Total & 199 & 66 & 33,2 & & & \\
\hline Raça & & & & & & 0,043 \\
\hline Branca & 152 & 45 & 29,6 & 1,00 & - & \\
\hline Não branca & 46 & 21 & 45,7 & 1,54 & {$[1,03 ; 2,30]$} & \\
\hline Total & 198 & 66 & 33,3 & & & \\
\hline Peso ao nascer & & & & & & 0,879 \\
\hline Adequado & 124 & 42 & 33,9 & 1,00 & - & \\
\hline Baixo & 63 & 21 & 33,3 & 0,98 & {$[0,64 ; 1,51]$} & \\
\hline Excesso & 7 & 3 & 42,9 & 1,27 & {$[0,52 ; 3,09]$} & \\
\hline Total & 194 & 66 & 34,0 & & & \\
\hline Tempo gestação & & & & & & 0,391 \\
\hline Prematuro & 27 & 11 & 40,7 & 1,00 & - & \\
\hline Não prematuro & 171 & 55 & 32,2 & 1,26 & {$[0,76 ; 2,09]$} & \\
\hline Total & 197 & 66 & 33,5 & & & \\
\hline Tempo amamentação & & & & & & 0,003 \\
\hline 6 meses ou mais & 129 & 34 & 26,4 & 1,00 & - & \\
\hline Inferior a 6 meses & 51 & 21 & 41,2 & 1,56 & {$[1,01 ; 2,42]$} & \\
\hline Não amamentou & 17 & 11 & 64,7 & 2,45 & {$[1,56 ; 3,87]$} & \\
\hline Total & 197 & 66 & 33,5 & & & \\
\hline Atividade física* & & & & & & 0,693 \\
\hline Não pratica & 60 & 19 & 31,7 & 1,00 & - & \\
\hline Pratica & 136 & 47 & 34,6 & 1,09 & {$[0,70 ; 1,69]$} & \\
\hline Total & 196 & 66 & 33,7 & & & \\
\hline Horas TV/diária & & & & & & 0,948 \\
\hline Até 1 hora & 58 & 20 & 34,5 & 1,00 & - & \\
\hline Até 2 horas & 63 & 21 & 33,3 & 0,97 & {$[0,59 ; 1,59]$} & \\
\hline Até 3 horas & 49 & 17 & 34,7 & 1,01 & {$[0,60 ; 1,70]$} & \\
\hline Acima de 3 horas & 28 & 8 & 28,6 & 0,83 & {$[0,42 ; 1,65]$} & \\
\hline Total & 198 & 66 & 33,3 & & & \\
\hline Consumo de fast food & & & & & & 0,615 \\
\hline Não & 97 & 34 & 35,1 & 1,00 & - & \\
\hline Sim & 101 & 32 & 31,6 & 0,90 & {$[0,61 ; 1,34]$} & \\
\hline Total & 198 & 66 & 33,3 & & & \\
\hline Escolaridade da mãe & & & & & & 0,744 \\
\hline Fundamental & 19 & 6 & 31,6 & 1,00 & - & \\
\hline Médio & 85 & 31 & 36,5 & 1,15 & {$[0,56 ; 2,37]$} & \\
\hline Superior & 93 & 29 & 31,2 & 0,99 & {$[0,48 ; 2,05]$} & \\
\hline Total & 197 & 66 & 33,5 & & & \\
\hline Peso da mãe & & & & & & 0,319 \\
\hline Normal & 121 & 36 & 29,8 & 1,00 & - & \\
\hline Baixo peso & 4 & 1 & 25,0 & 0,84 & {$[0,15 ; 4,71]$} & \\
\hline Excesso & 67 & 27 & 40,3 & 1,35 & {$[0,91 ; 2,02]$} & \\
\hline Total & 192 & 64 & 33,3 & & & \\
\hline Renda familiar & & & & & & 0,167 \\
\hline Até $\mathrm{R} \$ 1.000,00$ & 23 & 9 & 39,1 & 1,00 & - & \\
\hline Entre $R \$ 1.000,00$ e $2.000,00$ & 58 & 24 & 41,4 & 1,06 & {$[0,58 ; 1,92]$} & \\
\hline Entre $\mathrm{R} \$ 2.000,00$ e $4.000,00$ & 118 & 33 & 28,0 & 0,71 & {$[0,40 ; 1,29]$} & \\
\hline Total & 199 & 66 & 33,2 & & & \\
\hline
\end{tabular}

${ }^{*}$ Foi considerada como prática de atividade física a realização de qualquer atividade esportiva exercida regularmente, no mínimo, três vezes por semana, com pelo menos 1 hora de duração por dia 
Tabela 3. Análise multivariada (modelo de Poisson com variâncias robustas) dos fatores relacionados ao excesso de peso e à pressão arterial alterada de crianças com idade entre 8 e 10 anos das escolas da rede privada de Divinópolis/MG

\begin{tabular}{|c|c|c|c|c|c|c|}
\hline \multirow{2}{*}{ Variável } & \multicolumn{3}{|c|}{ Excesso de peso } & \multicolumn{3}{|c|}{ Pressão alterada } \\
\hline & $\mathbf{R P}$ & (IC95\%) & Valor $\mathbf{p}$ & $\mathbf{R P}$ & (IC95\%) & Valor $\mathbf{p}$ \\
\hline \multicolumn{7}{|l|}{ Sexo } \\
\hline Feminino & 1,00 & - & & & & \\
\hline Masculino & 2,05 & {$[1,36 ; 3,10]$} & 0,001 & & & \\
\hline \multicolumn{7}{|l|}{ Peso da mãe } \\
\hline Normal & 1,00 & - & & & & \\
\hline Baixo peso & 3,08 & {$[1,23 ; 7,70]$} & 0,016 & & & \\
\hline Excesso & 1,63 & {$[1,12 ; 2,36]$} & 0,010 & & & \\
\hline \multicolumn{7}{|c|}{ Pressão arterial da criança } \\
\hline Normal & 1,00 & - & & & & \\
\hline Alterada & 2,61 & {$[1,78 ; 3,81]$} & 0,000 & & & \\
\hline \multicolumn{7}{|l|}{ Raça } \\
\hline Branca & & & & 1,00 & - & \\
\hline Não branca & & & & 1,57 & {$[1,08 ; 2,27]$} & 0,017 \\
\hline \multicolumn{7}{|l|}{ Tempo amamentação } \\
\hline 6 meses ou mais & & & & 1,00 & - & \\
\hline Inferior a 6 meses & & & & 1,43 & {$[0,95 ; 2,17]$} & 0,089 \\
\hline Não amamentou & & & & 1,87 & {$[1,22 ; 2,86]$} & 0,004 \\
\hline \multicolumn{7}{|c|}{ Excesso de peso da criança } \\
\hline Normal & & & & 1,00 & - & \\
\hline Excesso de peso & & & & 2,53 & {$[1,71 ; 3,73]$} & $<0,001$ \\
\hline
\end{tabular}

Análise multivariada dos fatores associados ao peso corpóreo e à pressão arterial

Considerando como desfecho a presença de excesso de peso, foram incluídas no modelo multivariado as variáveis que apresentaram valor-p $<0,20$ na análise bivariada (idade, sexo, tempo de amamentação, horas na frente da TV, peso da mãe e $\mathrm{PA}$ ). Nesta análise, os fatores associados à maior prevalência de excesso de peso foram: sexo masculino, baixo peso ou excesso de peso da mãe e PA alterada (Tabela 3). Observou-se que a prevalência de excesso de peso foi de 2,05 vezes entre crianças do sexo masculino, se comparadas às do sexo feminino (podendo variar entre 1,36 e 3,10 na população). Já as crianças cujas mães tinham baixo peso tiveram uma probabilidade de 3,08 vezes de apresentar excesso de peso do que aquelas cujas mães tinham peso normal. Quando a mãe apresentava excesso de peso, a RP foi de 1,63. Por fim, crianças com PA alterada tiveram uma probabilidade de 2,61 vezes de ocorrência de excesso de peso, quando comparadas às crianças com pressão normal.

Para o desfecho da PA alterada, foram incluídas no modelo multivariado as variáveis raça, tempo de amamentação, renda familiar e excesso de peso da criança. Os fatores relacionados à maior prevalência de pressão alterada foram: raça não branca, não ter amamentado e presença de excesso de peso (Tabela 3). A probabilidade de pressão alterada foi de 1,57 vez entre crianças da raça não branca, se comparadas às de raça branca. As crianças que não foram amamentadas tiveram probabilidade de pressão elevada de 1,87 vez em relação àquelas que tiveram amamentação por seis meses ou mais. Já as crianças com excesso de peso tiveram probabilidade de 2,53 vezes de apresentarem pressão elevada do que aquelas com peso normal.

\section{DISCUSSÃO}

Dados na literatura têm demonstrado que níveis de PA alterados estão fortemente relacionados à presença de sobrepeso e de obesidade ${ }^{2,5,7,12}$. Essa importante associação também pode ser observada em crianças, o que aumenta a prevalência de doenças cardiovasculares durante a infância e, principalmente, durante a fase adulta ${ }^{17}$. Para minimizar a prevalência e as consequências do sobrepeso/obesidade e da HAS, é necessário conhecer os fatores associados a essas alterações para que medidas efetivas de prevenção possam ser adotadas. Como os fatores relacionados ao sobrepeso/obesidade e à HAS podem ser diferentes, dependendo da região do país, estudos locais são relevantes, pois podem contribuir para a adoção de medidas de prevenção mais eficazes.

Na cidade de Divinópolis/MG, a prevalência de sobrepeso e de obesidade, avaliada em crianças matriculadas em escolas públicas, com idade entre 6 e 14 anos, foi de 10,7 e 6,43\%, respectivamente ${ }^{11}$. Em nosso estudo, a prevalência de sobrepeso e de obesidade observada entre crianças de escolas privadas foi mais elevada (19,1 e 14,1\%, respectivamente), reforçando a hipótese de que, em países em desenvolvimento, crianças de classes econômicas mais altas apresentam maiores predisposições para desenvolver sobrepeso/obesidade ${ }^{12}$. Esse fato parece ter relação 
direta com o maior poder aquisitivo das famílias, que devido ao uso de equipamentos modernos e tecnológicos podem realizar atividades cotidianas com menor gasto calórico. Além disso, o consumo de alimentos mais calóricos é maior entre famílias de melhor renda, o que também pode ter relação com a maior prevalência de sobrepeso/obesidade entre as crianças ${ }^{12}$.

A relação entre o gênero e a prevalência de sobrepeso/obesidade é controversa. Alguns autores observaram em seus estudos uma associação significativa entre esses dois fatores ${ }^{18,19}$, enquanto outros não ${ }^{11,20}$. Dentre os trabalhos que sugerem haver uma relação entre sexo e excesso de peso corpóreo, alguns encontraram essa maior prevalência entre meninos ${ }^{18}$, como em nosso estudo, e outros, entre meninas ${ }^{19}$. Dessa forma, é possível que a relação entre o sexo e a prevalência de sobrepeso/obesidade esteja relacionada à região estudada e, consequentemente, ao estilo de vida adotado pelas crianças.

Por serem doenças de caráter multifatorial, tanto fatores genéticos como ambientais podem estar relacionados ao sobrepeso e à obesidade ${ }^{12,21}$. Reforçando a participação desses fatores no desenvolvimento do sobrepeso/obesidade, em nosso estudo foi encontrada uma associação positiva entre o peso corpóreo da mãe e o peso dos filhos. Crianças de mães com excesso de peso corpóreo apresentaram maior prevalência de sobrepeso/obesidade.

Da mesma forma, crianças que passam mais de 3 horas diárias na frente da TV ou do computador também apresentaram maior prevalência para excesso de peso.

O aspecto genético no desenvolvimento da obesidade está bem descrito na literatura ${ }^{22,23}$ e é respaldado por trabalhos que demonstram que o risco de obesidade entre as crianças aumenta quando um ou os dois pais apresentam sobrepeso/obesidade ${ }^{23,24}$. Porém, além do fator genético, a questão comportamental não pode ser ignorada, mesmo que esta seja condicionada geneticamente. Assim, o sedentarismo e o consumo de dieta hipercalórica são fatores que predispõem ao acúmulo de gordura corpóreo, e esses hábitos, quando familiares, podem ser repetidos pelas crianças ${ }^{25}$.

Dessa forma, é importante que os pais se reconheçam como exemplos para seus filhos e que adotem hábitos saudáveis, minimizando, assim, a tendência de sobrepeso/obesidade nas crianças e suas consequências.

Apesar dessa constatação, em nosso estudo, o consumo de fast food não se mostrou significativamente associado ao excesso de peso corpóreo. Porém, ao se analisar os aspectos vinculados a alterações na dieta, a obesidade pode ser decorrente do aumento da ingestão energética, da elevação quantitativa do consumo de alimentos, ou de ambos ${ }^{26}$. Assim, outros fatores relacionados ao consumo de fast food, por exemplo a quantidade consumida, também precisam ser considerados.
Também foi encontrada uma associação positiva entre mães de baixo peso e presença de sobrepeso/obesidade nas crianças. Porém, como nossa amostra de mães de baixo peso foi pequena, esse resultado precisa ser confirmado.

Diversas doenças, mais evidentes em adultos com excesso de peso corpóreo, já podem ser observada em faixas etárias menores, como dislipidemia e diabetes mellitus tipo $2^{2-5}$. Além dessas alterações, a HAS, que também é uma doença relacionada ao sobrepeso/obesidade, chama a atenção por ser um importante fator de risco para doenças cardiovasculares ${ }^{6,17}$.

O excesso de peso é um dos principais determinantes de alterações na PA de crianças, sobretudo a partir dos 5 anos de idade, havendo uma relação direta entre o IMC e os níveis de $\mathrm{PA}^{5}$. Corroborando esses dados, em nosso estudo foi observada uma forte relação entre presença de sobrepeso/obesidade e níveis pressóricos alterados.

Neste trabalho, os percentis de PA avaliados como limítrofe também foram considerados, uma vez que crianças com níveis de PA mais elevados, mesmo que dentro de limites considerados normais, tendem a manter uma PA mais elevada, apresentando maior probabilidade de se tornar um adulto hipertenso ${ }^{5}$. Além disso, a PA limítrofe também está associada a um aumento de risco cardiovascular ${ }^{27}$.

Os dados obtidos relativos aos níveis pressóricos das crianças são inéditos no município de Divinópolis/MG e se mostram preocupantes. Apesar da HAS em crianças não se apresentar como fator de risco para eventos cardiovasculares na infância, estes podem ser desencadeados após década de vida ou mesmo mais precocemente ${ }^{5}$.

Vários estudos epidemiológicos têm mostrado um aumento da prevalência de HAS na faixa etária pediátrica ${ }^{6,17}$. No entanto, os fatores associados a essa alteração ainda estão sendo investigados e a elucidação deles pode favorecer a adoção de novas medidas preventivas.

Em nosso trabalho, foi observada uma relação significativa entre a raça das crianças e os níveis pressóricos. Crianças não brancas apresentaram maior prevalência de PA alterada do que crianças classificadas como brancas. Essa relação é bem estabelecida entre adultos, onde pessoas da raça negra têm maior predisposição para a HAS do que pessoas brancas e apresentam maiores comprometimentos dos órgãos-alvo ${ }^{28}$. Entre crianças, apesar da associação entre raça e elevação dos níveis pressóricos ainda ser controversa, em nosso estudo foi encontrada uma relação significativa. Dessa forma, não é possível excluir a hipótese de que a elevação nos níveis pressóricos das crianças esteja relacionada a fatores genéticos.

Outro fator que mostrou relação com a alteração nos níveis pressóricos foi o tempo de aleitamento materno. Crianças que amamentaram no peito por um período superior a seis meses 
demonstraram menor prevalência de alterações na PA do que crianças que amamentaram por menos de seis meses.

Dentre os inúmeros benefícios produzidos pelo aleitamento materno, a longo prazo destaca-se a prevenção dos diversos fatores de riscos cardiovasculares, como obesidade, dislipidemias, diabetes e $\mathrm{HAS}^{29}$. Com relação ao efeito do aleitamento materno na prevenção HAS, alguns trabalhos têm sugerido que o efeito protetor da amamentação esteja relacionado ao baixo teor de sódio do leite materno em relação aos alimentos industrializados, à presença de ácidos graxos insaturados de cadeia longa no leite materno e à possibilidade de prevenção do sobrepeso/obesidade ${ }^{30}$. Mesmo não havendo um consenso sobre os mecanismos implicados no efeito protetor do aleitamento sobre os níveis pressóricos das crianças, não se pode descartar a hipótese de que a elevação da PA tem origem, pelo menos em parte, na condição nutricional precoce e que o aleitamento materno exerce um efeito protetor contra a HAS.

O sobrepeso/obesidade e a HAS são doenças crônicas, com múltiplas causas e consequências, e, uma vez diagnosticadas, representam desafios para os profissionais da saúde. O tratamento dessas doenças envolve, além de medicamentos, mudanças na alimentação e de comportamento, que, se não forem adotados, podem favorecer a progressão da doença. Dessa forma, a adoção de medidas de prevenção tem sido a melhor estratégia no controle do sobrepeso/obesidade e de HAS.

Estudos epidemiológicos como este têm grande importância, pois trazem informações valiosas sobre a saúde das crianças e alertam para as principais características de risco. É importante que pais e profissionais da saúde reconheçam esses fatores e atuem na prevenção do sobrepeso/obesidade e de HAS, estimulando hábitos saudáveis, especialmente em crianças mais predispostas a desenvolver essas doenças.

Considerando-se a sua característica epidemiológica, este estudo poderá proporcionar subsídios para que as Secretarias de Saúde e de Educação desenvolvam ações que atraiam a atenção da comunidade em geral e do poder público em relação aos cuidados com a saúde das crianças. Ações também poderão ser desenvolvidas nas escolas, conscientizando pais e responsáveis, visando à prevenção de doenças e à educação para a saúde.

\section{REFERÊNCIAS}

1. Instituto Brasileiro de Geografia e Estatística [Internet]. [citado em 2015 jun 10]. Disponível em: http://www.ibge.gov.br

2. Bray GA. Medical consequences of obesity. J Clin Endocrinol Metab. 2004;89(6):2583-9. http://dx.doi.org/10.1210/jc.2004-0535. PMid:15181027.

3. Daniels SR. Complications of obesity in children and adolescents. Int J Obes. 2009;33(Suppl 1):S60-5. http://dx.doi.org/10.1038/ijo.2009.20. PMid:19363511.

4. Oliveira CL, Mello MT, Cintra IP, Fisberg M. Obesidade e síndrome metabólica na infância e adolescência. Rev Nutr. 2004;17(2):237-45.

5. Sorof J, Daniels S. Obesity hypertension in children: a problem of epidemic proportions. Hypertension. 2002;40(4):441-7. http://dx.doi.org/10.1161/01. HYP.0000032940.33466.12. PMid:12364344.

6. Salgado CM, Carvalhaes JDA. Hipertensão arterial na infância. J Pediatr. 2003;79(Suppl 1):S115-24. http://dx.doi.org/10.1590/S0021-75572003000700013.

7. Ferreira JS, Aydos RD. Prevalência de hipertensão arterial em crianças e adolescentes obesos. Ciênc Saúde Coletiva. 2010;15(1):97-104. http:// dx.doi.org/10.1590/S1413-81232010000100015.

8. Sociedade Brasileira de Cardiologia-SBC, Sociedade Brasileira de Hipertensão-SBH, Sociedade Brasileira de Nefrologia-SBN. V Diretrizes Brasileiras de Hipertensão Arterial. Arq Bras Cardiol. 2007;89(3):e24-79. PMid:17906811.

9. Pinto SL, Silva RC, Priori SE, Assis AM, Pinto EJ. Prevalência de préhipertensão e de hipertensão arterial e avaliação de fatores associados em crianças e adolescentes de escolas públicas de Salvador, Bahia, Brasil. Cad Saude Publica. 2011;27(6):1065-76. http://dx.doi.org/10.1590/S0102311X2011000600004. PMid:21710004.

10. Brasil. Ministério da Saúde. Sistema de Informação [Internet]. [citado em 2015 jun 10]. Disponível em: http://www.datasus.gov.br

11. Pereira VV, Tiburcio JD, Alves SN, Silva ES. Avaliação de parasitoses intestinais, estado nutricional e indicadores sociais em alunos de quatro escolas do ensino fundamental público da cidade de Divinópolis-Minas Gerais, Brasil. Neotrop. Helminthol. 2010;4(2):149-57.

12. Silva GAP, Balaban G, Motta MEF. Prevalência de sobrepeso e obesidade em crianças e adolescentes de diferentes condições socioeconômicas. Rev Bras Saude Mater Infant. 2005;5(1):53-9. http://dx.doi.org/10.1590/S151938292005000100007.

13. Brasil. Ministério da Saúde. Secretaria de Políticas Públicas. Assistência pré-natal: manual técnico. Brasília, DF: Ministério da Saúde; 2000.

14. Sigulem DM, Devincenzi MU, Lessa AC. Diagnóstico do estado nutricional da criança e do adolescente. J Pediatr (Rio J). 2000;76(Suppl 3):S275-84. http://dx.doi.org/10.2223/JPED.164. PMid:14676905.

15. World Health Organization. Physical status: the use of and interpretation of anthropometry. Geneva; 1995. Report of a WHO Expert Committee.

16. Christofaro DG, Fernandes RA, Gerage AM, Alves MJ, Polito MD, Oliveira AR. Validation of the Omron HEM 742 blood pressure monitoring device in adolescents. Arq Bras Cardiol. 2009;92(1):10-5. http://dx.doi.org/10.1590/ S0066-782X2009000100003. PMid:19219259.

17. Luma GB, Spiotta RT. Hypertension in children and adolescents. Am Fam Physician. 2006;73(9):1558-68. PMid:16719248.

18. Balaban G, Silva GD. Prevalência de sobrepeso e obesidade em crianças e adolescentes de uma escola da rede privada de Recife. J Pediatr (Rio J). 2001;77(2):96-100. PMid:14647598.

19. Pelegrini A, Silva DAS, Petroski EL, Gaya ACA. Sobrepeso e obesidade em escolares brasileiros de sete a nove anos: dados do projeto Esporte Brasil. Rev Paul Pediatr. 2010;28(3):290-5.

20. Triches RM, Giugliani ERJ. Obesidade, práticas alimentares e conhecimentos de nutrição em escolares. Rev Saude Publica. 2005;39(4):541-7. http:// dx.doi.org/10.1590/S0034-89102005000400004. PMid:16113901. 
21. Tenorio AS, Cobayashi F. Obesidade infantil na percepção dos pais. Rev Paul Pediatr. 2011;29(4):634-9. http://dx.doi.org/10.1590/S010305822011000400025 .

22. Sorensen TIA. The genetics of obesity. Metabolism. 1995;44(9 Suppl 3):4-6. http://dx.doi.org/10.1016/0026-0495(95)90310-0. PMid:7674914.

23. Marques-Lopes I, Marti A, Moreno-Aliaga MJ, Martínez A. Genetics of obesity. Revista de Nutrição. 2004;17(3):327-38.

24. Ramos AMPP, Barros Fo ADA. Prevalência da obesidade em adolescentes de Bragança Paulista e sua relação com a obesidade dos pais. Arq Bras Endocrinol Metabol. 2003;47(6):663-8. http://dx.doi.org/10.1590/S000427302003000600007.

25. Mello ED, Luft VC, Meyer F. Obesidade infantil: como podemos ser eficazes. J Pediatr. 2004;80(3):173-82. http://dx.doi.org/10.2223/JPED.1180.

26. Mendonça CP, Anjos LA. Aspectos das práticas alimentares e da atividade física como determinantes do crescimento do sobrepeso/obesidade no Brasil. Cad Saude Publica. 2004;20(3):698-709. http://dx.doi.org/10.1590/ S0102-311X2004000300006. PMid:15263980.
27. Vasan RS, Larson MG, Leip EP, Kannel WB, Levy D. Assessment of frequency of progression to hypertension in non-hypertensive participants in the Framingham Heart Study: a cohort study. Lancet. 2001;358(9294):1682-6. http://dx.doi.org/10.1016/S0140-6736(01)06710-1. PMid:11728544.

28. Hertz RP, Unger AN, Cornell JA, Saunders E. Racial disparities in hypertension prevalence, awareness, and management. Arch Intern Med. 2005;165(18):2098-104. http://dx.doi.org/10.1001/archinte.165.18.2098. PMid:16216999.

29. Fewtrell MS. The long-term benefits of having been breast-fed. Curr Paediatr. 2004;14(2):97-103. http://dx.doi.org/10.1016/j.cupe.2003.11.010.

30. Martin RM, Ness AR, Gunnell D, Emmett P, Davey Smith G, ALSPAC Study Team. Does breast-feeding in infancy lower blood pressure in childhood? The Avon longitudinal study of parents and children. Circulation. 2004;109(10):1259-66. http://dx.doi.org/10.1161/01.CIR.0000118468.76447. CE. PMid:14993142.

Recebido em: Out. 24, 2014

Aprovado em: Ago. 25, 2015 